\title{
SMG-8 and SMG-9, two novel subunits of the SMG-1 complex, regulate remodeling of the mRNA surveillance complex during nonsense-mediated mRNA decay
}

\begin{abstract}
Akio Yamashita, ${ }^{1,2,8,12}$ Natsuko Izumi, ${ }^{1,8}$ Isao Kashima, ${ }^{1,9}$ Tetsuo Ohnishi, ${ }^{1,10}{ }^{1,}$ Bonnie Saari, ${ }^{3}$ Yukiko Katsuhata, ${ }^{1,4}$ Reiko Muramatsu, ${ }^{1,2}$ Tomoko Morita, ${ }^{1}$ Akihiro Iwamatsu, ${ }^{5}$ Takahisa Hachiya, ${ }^{6}$ Rie Kurata, ${ }^{1}$ Hisashi Hirano, ${ }^{7}$ Philip Anderson, ${ }^{3}$ and Shigeo Ohno ${ }^{1,11}$

${ }^{1}$ Department of Molecular Biology, Yokohama City University School of Medicine, Yokohama 236-0004, Japan; ${ }^{2}$ Precursory Research for Embryonic Science and Technology (PRESTO), Japan Science and Technology Agency, Kawaguchi 332-0012, Japan; ${ }^{3}$ Department of Genetics, University of Wisconsin at Madison, Madison, Wisconsin 53706, USA; ${ }^{4}$ Department of Obstetrics and Gynecology, Yokohama City University School of Medicine, Yokohama 236-0004, Japan; ${ }^{5}$ Protein Research Network Co. Ltd., Yokohama 236-0004, Japan; ${ }^{6}$ Ina Laboratory, Medical and Biological Laboratories Co. Ltd., Ina 396-0022, Japan; ${ }^{7}$ International Graduate School of Arts and Sciences, Yokohama City University, Yokohama 230-0045, Japan
\end{abstract}

\begin{abstract}
Nonsense-mediated mRNA decay (NMD) is a surveillance mechanism that detects and degrades mRNAs containing premature translation termination codons (PTCs). SMG-1 and Upf1 transiently form a surveillance complex termed "SURF" that includes eRF1 and eRF3 on post-spliced mRNAs during recognition of PTC. If an exon junction complex (EJC) exists downstream from the SURF complex, SMG-1 phosphorylates Upf1, the step that is a rate-limiting for NMD. We provide evidence of an association between the SURF complex and the ribosome in association with mRNPs, and we suggest that the SURF complex functions as a translation termination complex during NMD. We identified SMG-8 and SMG-9 as novel subunits of the SMG-1 complex. SMG-8 and SMG-9 suppress SMG-1 kinase activity in the isolated SMG-1 complex and are involved in NMD in both mammals and nematodes. SMG-8 recruits SMG-1 to the mRNA surveillance complex, and inactivation of SMG-8 induces accumulation of a ribosome:Upf1:eRF1:eRF3:EJC complex on mRNP, which physically bridges the ribosome and EJC through eRF1, eRF3, and Upf1. These results not only reveal the regulatory mechanism of SMG-1 kinase but also reveal the sequential remodeling of the ribosome:SURF complex to the predicted DECID (DECay InDucing) complex, a ribosome:SURF:EJC complex, as a mechanism of in vivo PTC discrimination.
\end{abstract}

[Keywords: NMD; mRNA surveillance; UPF1; SMG-1; PIKK; translation termination; mRNP remodeling] Supplemental material is available at http://www.genesdev.org.

Received November 25, 2008; revised version accepted March 23, 2009.

Translation termination involves recognition of a stop codon, followed by release of peptidyl-tRNA and recycling of the ribosome. These processes have been implicated in the recognition of premature termination codons (PTCs) during nonsense-mediated mRNA decay (NMD), a surveillance mechanism that ensures cellular mRNA quality. PTCs are generated by one-fourth of all mutations causing human genetic disease, and cancers often

\footnotetext{
${ }^{8}$ These authors contributed equally to this work.

Present addresses: ${ }^{9}$ Max-Planck-Institute for Developmental Biology, D-72076 Tübingen, Germany; ${ }^{10}$ Laboratory for Molecular Psychiatry, Brain Science Institute, RIKEN, Wako 351-0198, Japan.

Corresponding authors.

${ }_{11}^{11}$-MAIL ohnos@med.yokohama-cu.ac.jp; FAX 81-45-785-4140.

${ }^{12}$ E-MAIL yamasita@yokohama-cu.ac.jp; FAX 81-45-785-4140.

Article is online at http://www.genesdev.org/cgi/doi/10.1101/gad.1767209.
}

exhibit abnormal transcription and mRNA processing (Holbrook et al. 2004; Jaillon et al. 2008). Thus, NMD likely protects cells from accumulation of potentially harmful C-terminally truncated proteins encoded by PTC-containing mRNAs (Conti and Izaurralde 2005; Amrani et al. 2006; Kuzmiak and Maquat 2006; Chang et al. 2007). In some cases, truncated proteins retain at least some of their normal function, and the selective inhibition of NMD provides a novel strategy to rescue the mutant phenotype of certain PTC-related mutations (Pulak and Anderson 1993; Cali and Anderson 1998; Usuki et al. 2004, 2006; Welch et al. 2007).

PTCs are recognized during NMD by successive remodeling of a "surveillance complex," a putative substance that discriminates PTC-containing mRNAs from normal mRNAs and triggers degradation of PTC-containing 
mRNAs. In a current model of PTC recognition in mammals, a surveillance complex termed "SURF" because it contains SMG-1, Upf1, eRF1, and eRF3 assembles first during translation termination. If an exon junction complex (EJC) is located $50-55$ or more nucleotides downstream from the termination codon, the SURF complex physically associates with the downstream EJC through Upf 2 and Upf3 on post-spliced mRNPs that also contain CBP20, a marker of the initial round of translation. A proper association between SURF and the EJC guarantees that the termination codon is recognized as a PTC, and this "judgment" is followed by phosphorylation of Upf1 by its kinase SMG-1. Upf1 phosphorylation is a ratelimiting step during NMD (Yamashita et al. 2001, 2005; Ohnishi et al. 2003; Kashima et al. 2006).

The above model predicts that PTC recognition occurs during ribosome-mediated mRNA translation, that remodeling of the surveillance complex occurs during termination, and that SMG-1 kinase activity is tightly regulated during these processes. The physical association between SURF and the EJC has not been demonstrated, and important aspects of the model have not been demonstrated.

SMG-1 is a member of the family of phosphatidylinositol 3-kinase-related protein kinases (PIKKs) that play central roles in cell growth and stress response pathways. In addition to NMD, SMG-1 has been shown to be involved in genotoxic and oxidative stress response pathways (Brumbaugh et al. 2004; Gehen et al. 2008) and TNF $\alpha$-induced apoptosis (Oliveira et al. 2008) as other members of the PIKK family, ATM (ataxia telangiectagia mutated) and ATR (ATM- and Rad3-related) (Shiloh 2003). Although these observations raise the question as to the similarity and difference between SMG-1 and other PIKKs, the nature and the regulatory mechanism of SMG-1 kinase remains poorly understood.

We report here identification of two novel NMD regulators, SMG-8 and SMG-9. Both are tightly associated with SMG-1 to form the SMG-1 complex, and both are conserved among metazoa. SMG-8 and SMG-9 are novel components of the SURF complex, which associates with ribosomes on mRNPs during translation termination and NMD. We demonstrate that SMG-8 and SMG-9 suppress SMG-1 kinase activity in the isolated SMG-1 complex, suggesting that they regulate SMG-1 during remodeling of the surveillance complex. SMG-8 is additionally required to recruit SMG-1 to the surveillance complex where SMG-1 kinase should be activated. These two regulatory mechanisms can explain how SMG-1 kinase is suppressed until SURF associates with EJC. Moreover, depletion of SMG-8 causes the accumulation of the ribosome:Upf1:eRF1:eRF3:EJC complex on mRNPs. This indicated not only the abortive interaction between the ribosome:Upf1:eRF1:eRF3 complex and EJC in the absence of the SMG-1 complex but also the importance of the interaction between the SMG-1 complex and EJC. Both of these two independent protein-protein interactions are likely required for the formation of the previously predicted DECID complex, which connects the PTC with the exon-exon junction to distinguish termi- nation codons as premature. Our results identify mechanisms important not only for distinguishing PTCs from normal termination codons but also for aspects of normal translation termination.

\section{Results}

Identification of novel components of the SMG-1 complex

Gel filtration column chromatography indicated that SMG-1 elutes more quickly than expected from its molecular weight, suggesting that it forms a stable protein complex (data not shown). We therefore immunoprecipitated SMG-1 from HeLa TetOff cell lysates and established that SMG-1 copurifies with at least two proteins, pp130 and pp60. As shown in Figure 1A, when SMG-1 immunoprecipitates were incubated with ${ }^{32} \mathrm{P} \gamma$-ATP in a buffer favorable for kinases, ${ }^{32} \mathrm{P}$ was incorporated into the 130 $\mathrm{kDa}(\mathrm{pp} 130)$ and 60-kDa (pp60) bands in addition to being incorporated into SMG-1 itself (pp400) by autophosphorylation. We obtained similar results using three independent anti-SMG-1 antibodies (Fig. 1A; data not shown). These results suggest that SMG-1 forms a large complex, which we term "SMG1C" (SMG-1 complex), and that both pp130 and pp60 are components of SMG1C. Similar immunoprecipitation/autophosphorylation experiments using cell lysates fractionated by anion exchange column chromatography confirmed the tight association of these three proteins and established that this association was not shared with other members of the PIKK family kinases (Supplemental Fig. 1). The sensitivities of in vitro pp130 and pp60 phosphorylation to $\mathrm{Mn}^{2+}$ ions, wortmannin, and caffeine suggest that phosphorylation of pp130 and pp60 is mediated by the intrinsic kinase activity of SMG-1 (Supplemental Fig. 2).

Silver-stained proteins that copurify with SMG-1 following immunoprecipitation with our SMG-1-N antibody are shown in Figure 1B. Both pp130 and pp60, which comigrates with IgG signals, are evident. Peptide mass fingerprinting analysis of these bands identified pp130 and pp60 as proteins FLJ23205 and FLJ12886, respectively. Because the $75-\mathrm{kDa}$ band did not copurify with SMG-1 using other anti-SMG-1 antibodies, it is most likely a cross-reacting protein revealed only by the SMG-1-N antibody. FLJ23205 is a previously uncharacterized putative protein of 991 amino acids (Fig. 1C) whose gene maps to human chromosome 17q22. The predicted protein does not contain any significant protein motifs using public databases and available motif searching programs. Blast searches revealed putative orthologs in many metazoan genomes but not in the yeast and Arabidopsis thaliana genome. Alignment of pp130 across species defined two highly conserved regions (Fig. 1C; Supplemental Fig. 3). Because of its conservation and because the Caenorhabditis elegans ortholog of pp130 is involved in nematode NMD (see below), we refer to pp130 as "SMG-8." FLJ12886 is a previously uncharacterized putative protein of 520 amino acids (Fig. 1C) whose gene maps to human chromosome 19q13.31. We 
A

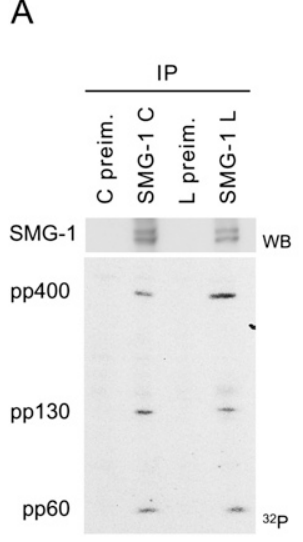

B

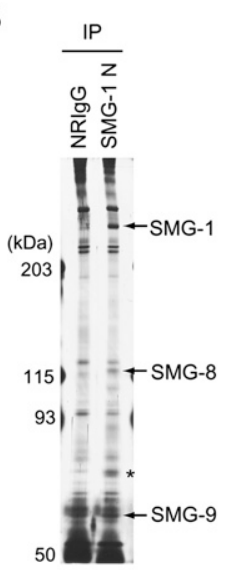

C

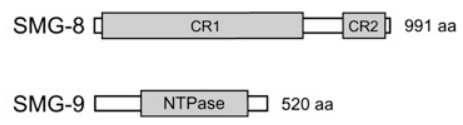

D

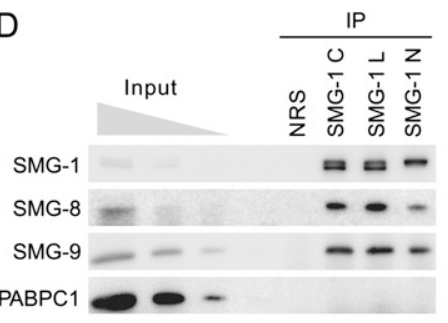

E
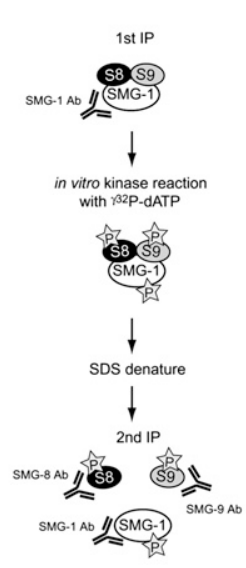

$\mathrm{F}$

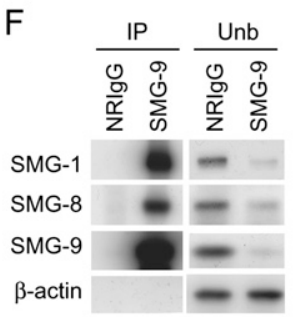

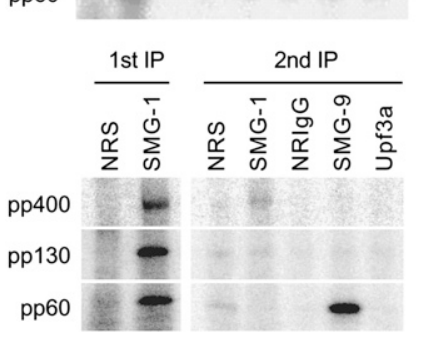

Figure 1. Identification of SMG-8 and SMG-9, subunits of the SMG-1 complex (SMG1C). (A) HeLa cell lysates were immunoprecipitated with anti-SMG-1 antiserum (C or L) (Yamashita et al. 2001) or with preimmune serum (C preim. or L preim.). In vitro kinase reactions were then performed without substrates. (B) HeLa cell extracts were subjected to immunoprecipitation with anti-SMG-1-N antibody (Yamashita et al. 2001) or normal rabbit IgG (NRIgG). Coprecipitated proteins were detected by silver staining following SDS-PAGE. The signals indicated by arrows were analyzed by mass spectrometry. Asterisk indicates the SMG-1-N antibody background precipitation. $(C)$ Schematic representation of SMG8 and SMG-9. Gray boxes indicate conserved regions (CR) among metazoans. ( $D)$ HeLa TetOff cell extract was subjected to immunoprecipitation with three different anti-SMG-1 antisera $(\mathrm{C}$, L, or $\mathrm{N})$ or NRS. Immunoprecipitates were probed with the antibodies indicated. (E) HeLa TetOff cell extract was subjected to immunoprecipitation with anti-SMG-1-C antiserum or NRS. In vitro kinase reactions were performed with recovered immunocomplexes. After the kinase reactions, complexes were denatured in 2\% SDS and then diluted 10-fold. Denatured proteins were subjected to the second immunoprecipitation with the antibodies indicated. Phosphorylated proteins were detected by autoradiography following SDSPAGE. Schematic representation of this experiment is illustrated on the left. (F) HeLa TetOff cell lysates were immunoprecipitated with antiSMG-9 antibody or NRIgG. Both immunoprecipitate (IP) and unbound fractions (Unb) were analyzed using the antibodies indicated. term the pp60 protein "SMG-9." The central region of SMG-9 is a putative NTPase domain (Fig. 1C; Supplemental Fig. 4). Like SMG-8, SMG-9 is conserved among metazoans but is not found in yeast and A. thaliana. To characterize SMG-8 and SMG-9, we raised antibodies against SMG-8 and SMG-9 (Supplemental Fig. 5) and investigated the presence of SMG-8 and SMG-9 in the SMG1C. Three different anti-SMG-1 antibodies specifically coimmunoprecipitated SMG-8 and SMG-9 with SMG-1 in the presence of RNase (Fig. 1D). Thus, both SMG-8 and SMG-9 form a complex with SMG-1. Tandem immunoprecipitation analysis further confirmed that SMG-8 and SMG-9 are pp130 and pp60, respectively. Following denaturation by $2 \%$ SDS and dilution to $0.2 \%$ SDS of anti-SMG-1 immune complexes that had been phosphorylated in vitro, the anti-SMG- 8 antibody recovered pp130 but not pp400 or pp60 (Fig. $1 \mathrm{E}$, top panel). Parallel experiments with the anti-SMG-9 antibody recovered pp60 but not pp400 or pp130 (Fig. $1 \mathrm{E}$, lower panel). Similarly, the anti-SMG-1 antibody recovered pp400 but not pp130 or pp60 (Fig. 1E). The anti-SMG-7 antibody, anti-SMG-5 antibody, antiUpf3a antibody, normal rabbit immunoglobulin G (NRIgG), and normal rabbit serum (NRS) failed to recover any signals (Fig. 1E; data not shown). To confirm the tight association of SMG-8 and SMG-9 with SMG-1, we performed immunoprecipitation and immunodepletion experiments using our anti-SMG-9 antibody. As shown in Figure 1F, SMG-1 and SMG-8 copurified with immunoprecipitated SMG-9. Intriguingly, under conditions that immunoprecipitate almost all SMG-9 from cell extracts, the majority of both SMG-1 and SMG-8 are coimmunoprecipitated (Fig. 1F, unbound fractions). The tight associations among the SMG1C components are also supported by the coimmunoprecipitation of SMG-8 and SMG-9 with Flag-SMG-1 in non-RNase-treated cytoplasmic fractions and in RNase-treated cytoplasmic and nuclear fractions (Supplemental Fig. 6). This is in contrast to Upf1 and Upf2, which are not coimmunoprecipitated in the nuclear fraction.

\section{SMG-8 directly regulates $S M G$-1 kinase activity}

SMG-1-mediated Upf1 phosphorylation is essential for NMD, suggesting that the kinase activity of SMG-1 is tightly regulated during remodeling of the surveillance complex (Yamashita et al. 2001; Ohnishi et al. 2003; Grimson et al. 2004; Kashima et al. 2006). The tight association of SMG-1 with SMG-8 and SMG-9 raises the possibility that they regulate SMG-1 kinase activity. To 
evaluate this possibility, we immunopurified the SMG1C from cells in which either SMG-8 or SMG-9 had been depleted by RNAi and investigated which proteins copurified with it and assessed the kinase activity of SMG1C. We used high stringency wash conditions that eliminated detectable Upf1 and SMG-7 from the immunoprecipitates (Morita et al. 2007). SMG-1 immunoprecipitates prepared from cells depleted of SMG-9 copurified with significantly reduced quantities of both SMG-9 and SMG-8 (Fig. 2A). On the other hand, SMG-1 immunoprecipitates prepared from cells depleted of SMG-8 copurified with unchanged amounts of SMG-9 (Fig. 2A). These results indicate that SMG-9 is required for association of SMG-1 with SMG-8. SMG-1 kinase activity using GST-

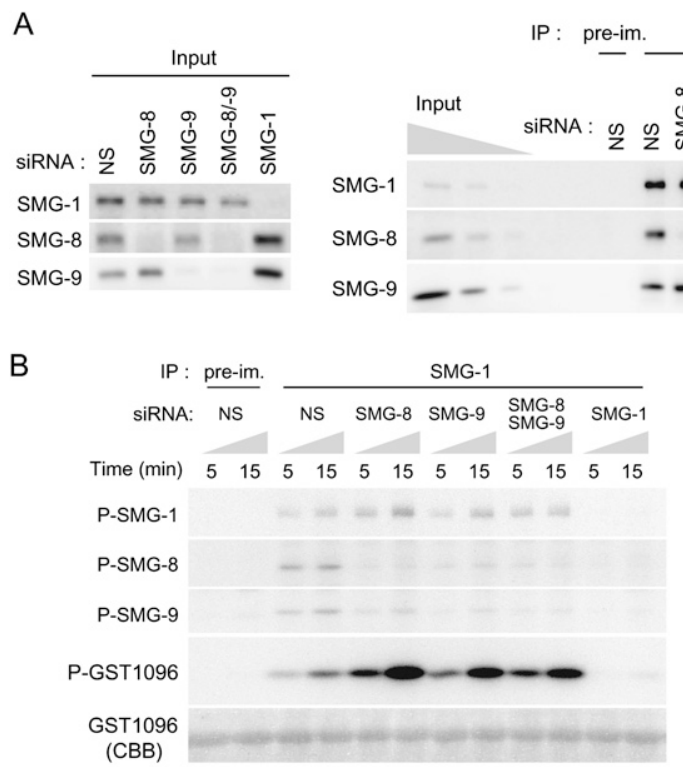

C

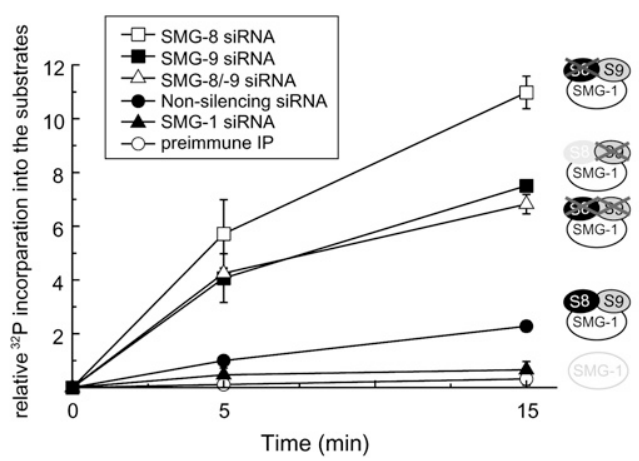

Figure 2. SMG-8 is an inhibitor of SMG-1 kinase. (A) HeLa TetOff cells were transfected with the siRNAs indicated. SMG-1 complexes were immunoprecipitated with anti-SMG-1-C antiserum and analyzed by Western blotting. (NS) Nonsilencing siRNA; (pre-im) preimmune serum. (B) In vitro kinase assay with SMG-1 immunoprecipitates as the kinase source and GSTS1096 (GST fusion peptide containing Ser1096 of Upf1) as the substrate. (C) Quantification of the phosphorylation level of GST-S1096 in vitro. Relative values against NS-treated control are shown. Mean values \pm SD from three independent experiments are shown.
Upf1-S1096 as a substrate (Yamashita et al. 2001) was greatly increased in SMG1Cs purified from cells depleted of SMG-8 (Fig. 2B,C). Thus, SMG-8 plays a role as an inhibitor of SMG-1 kinase activity in the isolated SMG-1 complex. SMG-1 kinase activity purified from cells depleted of both SMG-8 and SMG-9 is reduced in comparison with SMG1Cs that contain SMG-9 but not SMG8 (Fig. 2B,C), suggesting that SMG-9 is a positive regulator of SMG-1 kinase activity. However, because SMG-9 is required for the association of SMG-1 and SMG-8, SMG-9 probably functions together with SMG-8 as an inhibitor of SMG-1 kinase activity. We obtained similar results using purified full-length HA-Upf1 as the SMG-1 substrate (data not shown). Additionally, we observed that the SMG-1-mediated phosphorylation of SMG-9 was decreased in cells depleted of SMG-8 in vitro (Fig. 2B).

\section{SMG-8 and SMG-9 are involved in the ribosome:SURF complex}

Tight association of SMG-8 and SMG-9 with SMG-1 suggests that they function as part of the mRNA surveillance complex. To evaluate this possibility, we employed a mutant Upf1 protein (Upf1-C126S) that loses the ability to bind to Upf2 and, hence, the EJC in vivo (Kashima et al. 2006). Upf1-C126S freezes the surveillance complex, resulting in accumulation of the SURF complex (Kashima et al. 2006). HA-tagged wild-type Upf1 or HA-Upf1-C126S were expressed in HeLa TetOff cells and immunopurified using an anti-HA affinity matrix in the presence of RNase. As expected, Upf1-C126S copurified with greater amounts of SMG-8, SMG-9, and SMG-1 compared with wild-type Upf1, and failed to copurify with Upf2 and Y14, an EJC component (Fig. 3A). These results strongly indicate that SMG-8 and SMG-9 are components of the SURF complex.

PTCs have been assumed to be recognized by ribosomes during translation termination (Belgrader et al. 1993), and mRNA surveillance complex expected to associate with ribosome but little direct evidence supports this. We therefore investigated whether ribosomal proteins associate with the Upf1-C126S complex in the presence of cycloheximide, which stabilizes $80 \mathrm{~S}$ ribosomes on mRNAs in vitro and prevents their dissociation during immunoprecipitation (Baliga et al. 1969). Upf1C126S copurified with increased amounts of ribosomal proteins rpL7a, rpL11, rpS3, and rpS6 and with SURF components eRF3 and SMG-1 compared with wild-type Upf1 (Fig. 3A,B). As expected, Upf1-C126S failed to precipitate Upf2 (Fig. 3B). These results suggest a physical association between the SURF complex and the ribosome. To confirm this, we investigated associations of ribosomal proteins with the Upf1 complex under the condition of Y14 depletion, which is known to cause accumulation of the SURF complex most likely via disruption of EJC (Kashima et al. 2006). We immunopurified endogenous Upf1 in the presence of RNase and cycloheximide using the mouse monoclonal anti-Upf1 antibody 5C3 (Supplemental Fig. 7). After immunoprecipitation, 
A

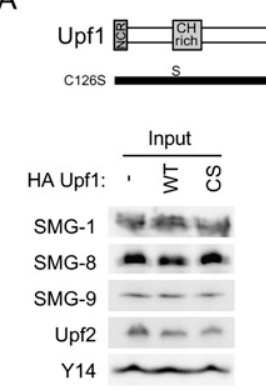

B

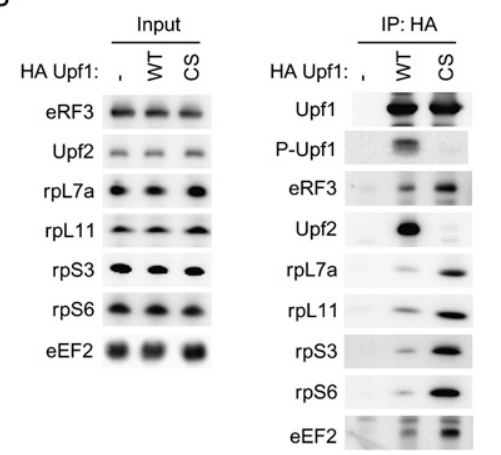

C

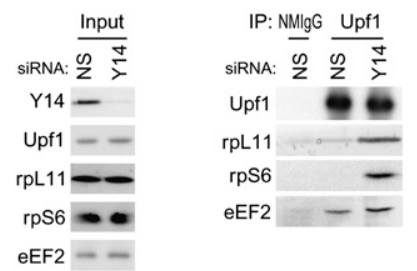

D

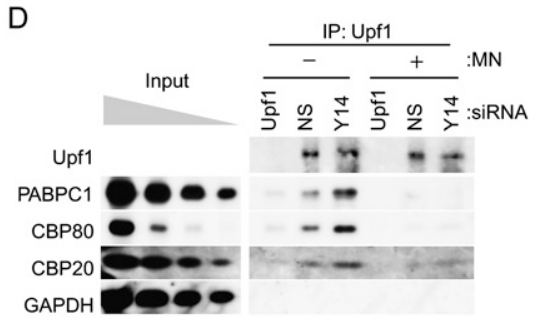

E

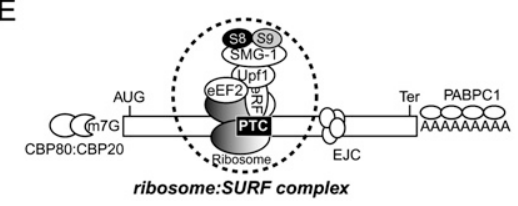

Figure 3. SMG-8 and SMG-9 are components of the mRNA surveillance complex. $(A, B)$ Schematic structure of the Upf1C126S mutant (A, top panel). HeLa TetOff cells were transfected with plasmids encoding wild-type HA-Upf1 (WT) or HAUpf1-C126S (CS). A minus sign (-) indicates empty vector. The cell extracts (input) were immunoprecipitated with antiHA affinity matrix under the conditions of RNase-treatment with $(B)$ or without $(A)$ cycloheximide. Eluted immunocomplexes were probed with the antibodies shown on the left. $(C, D)$ HeLa TetOff cells were transfected with siRNAs shown above the blots. The cell extracts (input) were immunoprecipitated (IP) with anti-Upf1 (5C3) antibody or with normal mouse IgG (NMIgG) in the presence of RNase and cycloheximide $(C)$ or in the presence of cycloheximide, and the presence or absence of micrococcal nuclease $(\mathrm{MN})(D)$. Input (the NS siRNAtreated cell extract equivalent to $10 \%$, $3.3 \%, 1.1 \%$, and $0.33 \%$ of the amount immunoprecipitated) and eluted immunoprecipitated fractions were analyzed by Western blotting with the antibodies indicated. $(E)$ Model illustrating the riboso-

me:SURF complex on mRNPs during PTC recognition. Details are described in the text. (eRF) eRF1:eRF3 complex; (EJC) exon junction complex; (AUG) start codon; (Ter) termination codon; (PTC) premature termination codon.

Upf1 immune complexes were eluted with the Upf1peptide antigen. As expected, ribosomal proteins rpL11 and rpS6 were concentrated in the immune complexes of Y14-depleted cell extracts (Fig. 3C). These results strongly suggest that the SURF complex physically associates with ribosomes. Additionally, we identified eukaryotic elongation factor 2 (eEF2) in immunoprecipitates of Upf1 (Supplemental Fig. 7), suggesting that eEF2 is involved in post-translation termination (Demeshkina et al. 2007). We therefore investigated whether eEF2 copurifies with the SURF complex. As expected, immunoprecipitation of Upf1-C126S or Upf1 from Y14-depleted extracts copurified with larger amounts of eEF2 than immunoprecipitation of wild-type Upf1 or Upf1 from control cells (Fig. 3B,C). These results indicate that eEF2 is also a component of the ribosome:SURF complex, although the accumulation of eEF2 was not as significant as that of ribosomal proteins.

We next tested whether the ribosome:SURF complex is associated with mRNPs. We immunopurified endogenous Upf1 from total cell extracts of HeLa TetOff cells that had been treated with nonsilencing (NS) or Y14silencing siRNAs in the presence of cycloheximide. Immunoprecipitates were incubated for $30 \mathrm{~min}$ at $25^{\circ} \mathrm{C}$ with or without micrococcal nuclease, after which Upf1containing complexes were eluted using the Upf1-peptide antigen. As shown in Figure 3D, significant enrichments of PABPC1, the cytoplasmic poly(A)-binding protein, CBP20, and CBP80, nuclear cap-binding proteins, were observed in Upf1 immunoprecipitates of Y14-depleted cell extracts. The association between Upf1 and PABPC1, CBP20, and CBP80 was significantly decreased by micrococcal nuclease treatment, confirming that the ribosome:SURF complex is formed on mRNPs (see Fig. 3E). Depletion of SMG-8 causes a ribosome:Upf1:eRF1:
eRF3:EJC complex to accumulate on mRNPS

Our current model suggests that the ribosome:SURF complex interacts with the EJC after recognition of a termination codon (Fig. 8, below). To clarify the role of SMG-8 and SMG-9 in this process, we investigated whether SMG-8 and SMG-9 associate with the EJC. We immunoprecipitated Y14 from RNase treated HeLaTetOff cell extracts and tested whether it copurifies with SMG-8, SMG-9, and other EJC- or NMD-related proteins. Our results demonstrate that SMG-8, SMG-9, SMG-1, eIF4A3, and Magoh, but not PABPC1 or $\beta$-actin, copurify with Y14 in the presence of RNase (Supplemental Fig. 8). Additionally, immunoprecipitation of SMG-8 or SMG-9 expressed exogenously following transfection copurified with endogenous EJC components (data not shown). These results indicate that SMG-8 and SMG-9 can associate with the EJC.

Because SMG-8 and SMG-9 associate with SMG-1 in the SURF complex and interact with the EJC (Fig. 3A; Supplemental Fig. 8), we investigated whether SMG-8 and SMG-9 influence the association of SMG-1 with Upf1 and/or the EJC. We immunoprecipitated endogenous SMG-1 from cell extracts that had been depleted of 
A

Figure 4. SMG-8 knockdown causes the accumulation of the complex containing ribosome, eRF1, eRF3, Upf1, and EJC on mRNPs. $(A-E)$ HeLa TetOff cells were transfected with the indicated siRNAs and the cell extracts were analyzed by Western blotting with the indicated antibody probes (left panel: input). The cell extracts were immunoprecipitated with anti-SMG-1-N antibody $(A)$, anti-SMG-1IP antibody (B), anti-Y14 (4C4) antibody $(C)$, anti-HA antibody (12CA5, used as a control IgG), or anti-Upf1 (5C3) antibody $(D, E)$ in the presence of RNase $(A-D)$ or in the absence/presence of micrococcal nuclease $(\mathrm{MN})(E)$. The cell extracts (left panel: input) and immunoprecipitates (right panel: IP) were analyzed by Western blotting with the antibodies shown on the left. (F) Schematic presentation of Upf1 complexes following SMG-8 siRNA treatment. SMG-8 knockdown leads to the accumulation of two distinct Upf1 complexes; SMG-1:SMG-9:Upf1 complex and ribosome:Upf1:eRF1:eRF3:EJC complex on mRNPs. Details are described in the text. (eRF) eRF1:eRF3 complex, (EJC) exon junction complex; (AUG) start codon; (Ter) termination codon, (PTC) premature termination codon.

B

C
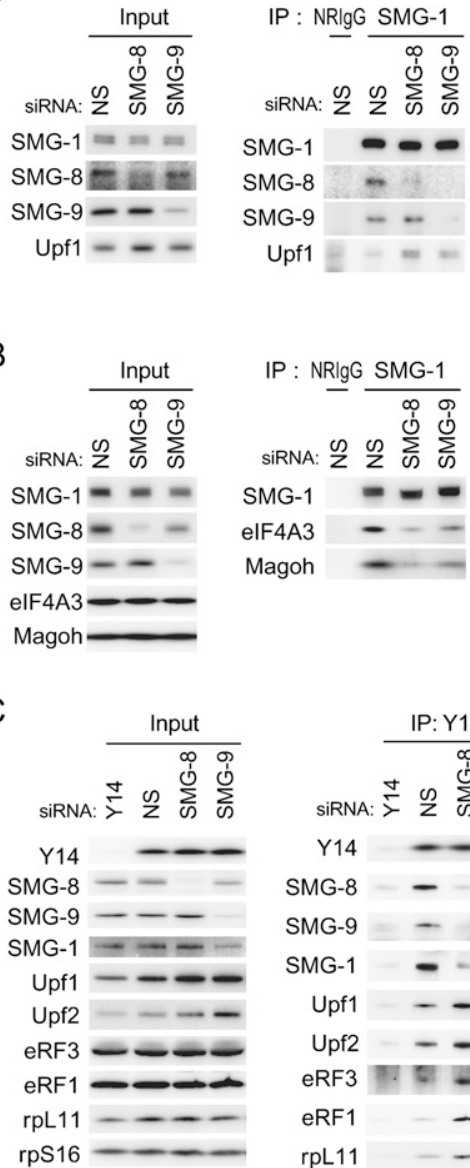

SMG-8 or SMG-9 in the presence of RNase and analyzed the amount of copurifying Upf1. Compared with a NS control, depletion of SMG-8 or SMG-9 slightly enhanced the amount of copurifying Upf1 (Fig. 4A). Similar results were observed following immunoprecipitation of exogenously expressed Flag-tagged SMG-1 (Supplemental Fig. 9). However, the amount of copurifying EJC components, eIF4A3 and Magoh, with SMG-1 was decreased in SMG8- or SMG-9-depleted cell extracts (Fig. 4B). These results suggest that SMG-8 and SMG-9 are involved in the association of SMG-1 with the EJC. To further evaluate this, we investigated protein components of the Y14 immunoprecipitates in HeLa-TetOff cell extracts depleted for SMG-8 or SMG-9 and in the presence of RNase. Similar to results described above, following immunoprecipitation of SMG-1, immunoprecipitation of Y14 copurified with significantly reduced quantities of SMG-1 and SMG-9 in SMG-8-depleted extracts (Fig. 4C).

The amount of Upf1 that copurifies with immunoprecipitated Y14 or SMG-1 increased in SMG-8-depleted cell extracts (Fig. 4A,C), although the association between SMG-1 and EJC decreased in the SMG-8 depleted condition (Fig. 4B,C). These results suggest that at least two Upf1-complexes exist in the absence of SMG-8: one containing SMG-1 and SMG-9, and another containing the
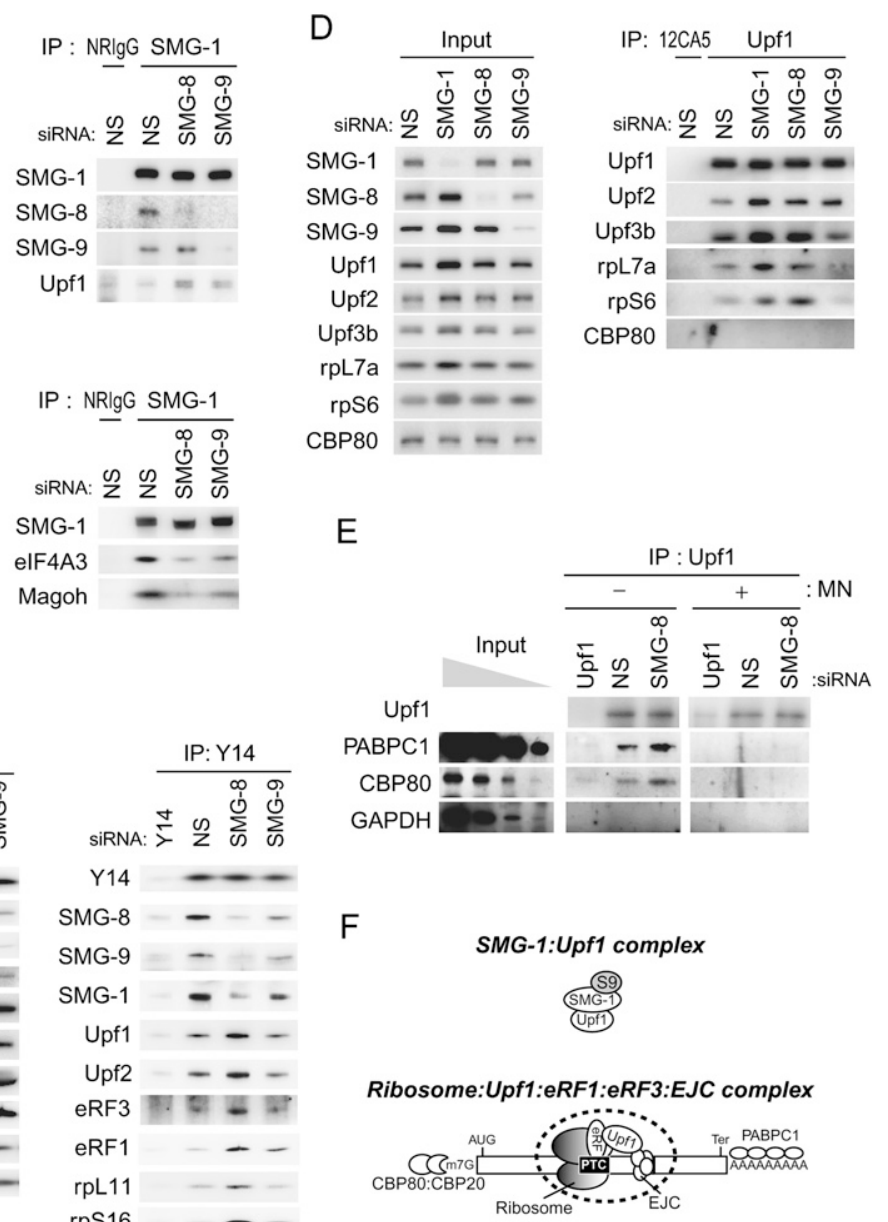

EJC but not SMG-1 and SMG-9. This suggests that a complex between the ribosome, Upf1, and the EJC forms in the absence of the SMG1C. To test this possibility, we investigated whether ribosomal proteins, eRF1, eRF3, and Upf2 copurify with Y14 from SMG-8-depleted cell extracts. Intriguingly, the ribosome:SURF components rpL11, rpS16, eRF1, eRF3, and Upf2 were enriched in Y14 immunoprecipitates (Fig. 4C). These results provide the first evidence for a physical interaction between the ribosome and the EJC, probably through Upf1. To further confirm this, we investigated the Upf1 immunoprecipitates in extracts of HeLa-TetOff cells treated with siRNAs targeting SMG-1, SMG-8, SMG-9, or NS siRNAs in the presence of RNase. Consistent with the results described above, increased amounts of ribosomal proteins rpL7a and rpS6 and EJC components Upf2 and Upf3b copurified with Upf1 in RNase-treated extracts depleted of SMG-8 or SMG-1 (Fig. 4D). We next investigated whether the ribosome:Upf1:eRF1:eRF3:EJC complex forms on mRNPs. We immunopurified endogenous Upf1 from extracts of HeLa TetOff cells depleted of Upf1 or SMG-8 and incubated the immunoprecipitates for 30 min at $25^{\circ} \mathrm{C}$ with or without micrococcal nuclease. We then eluted Upf1 with the Upf1-peptide antigen, and determined whether the mRNP markers PABPC1 and CBP80 
copurified with Upf1 in an RNA-dependent manner. As shown in Figure 4E, increased amounts of PABPCl and CBP80 copurify with the Upf1 complex in SMG-8-depleted cell extracts, but only in extracts not treated with micrococcal nuclease. These results indicate that depletion of SMG-8 causes accumulation of a ribosome:Upf1:eRF1: eRF3:EJC complex on mRNPs. Our model is depicted in Figure 4F.

SMG-9 siRNA treatment did not significantly alter the coprecipitation of analyzed proteins in the Y14 or Upf1 immunoprecipitates (Fig. 4C,D), except for decreasing association of SMG-1 and SMG-8 in the Y14 immunoprecipitates (Fig. 4B,C).

\section{SMG-8 is required for phosphorylation of Upf1} by SMG-1 in vivo

Although SMG-8 suppresses SMG-1 kinase activity in isolated SMG-1 complex (Fig. 2), accumulation of a ribosome:Upf1:eRF1:eRF3:EJC:mRNP complex that does not contain SMG-1 in SMG-8-depleted cell extracts suggests that SMG-8 additionally functions to recruit the SMG1C to ribosomes stalled at PTCs. We demonstrated previously that Upf1 phosphorylation occurs on

A

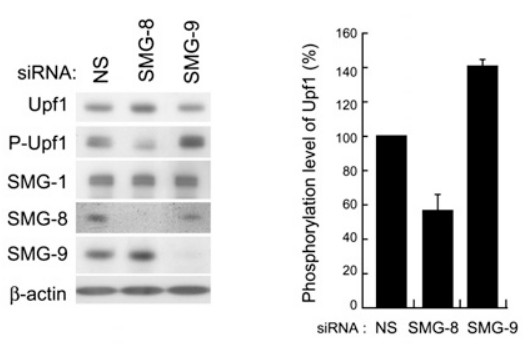

B

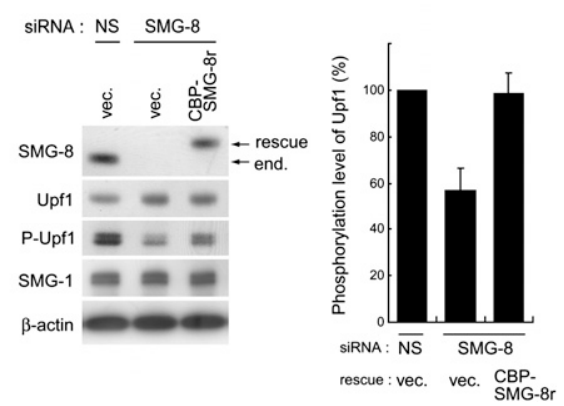

Figure 5. SMG-8 is required for SMG-1-mediated Upf1 phosphorylation in vivo. (A) HeLa TetOff cell lysates transfected with the siRNAs shown above the blots were analyzed by Western blotting with the antibody probes indicated. Anti-PUpf1 antibody recognizes phosphorylated Ser1078 and Ser1096 residues of Upfl. (B) HeLa TetOff cells were treated with the siRNA indicated above the blot and subsequently transfected with plasmid expressing CBP-SMG-8r harboring a silent mutation in the siRNA targeting site. Cell lysates were analyzed by Western blotting with the antibodies indicated and the relative values of phospho-Upf1, normalized to Upf1 signals, were plotted. $(A, B$, right panels) Mean values \pm SD from three independent experiments are shown. post-spliced mRNAs (Kashima et al. 2006). SMG-8 depletion, therefore, should reduce Upf1 phosphorylation in vivo. To test this, we inhibited expression of SMG-8 or SMG-9 in HeLa TetOff cells using siRNAs and evaluated phospho-Upf1 levels. As expected, SMG-8 knockdown reduced the amount of phospho-Upf1 (Fig. 5A). Similar results were obtained using a different siRNA targeted to SMG-8 (Supplemental Fig. 10). Coexpression of an siRNA-resistant SMG-8 restored phospho-Upf1 levels (Fig. 5B). In contrast, depletion of SMG-9 caused an increase in the amount of phospho-Upf1 (Fig. 5A).

\section{SMG-8 and SMG-9 are required for mammalian NMD}

As described above, SMG-8 knockdown caused defects in remodeling of the mRNA surveillance complex and reduced Upf1 phosphorylation in vivo. SMG-9 knockdown caused increased accumulation of phosho-Upfl. If these remodeling steps and the cycles of Upf1 phosphorylation are important for NMD, SMG-8 or SMG-9 knockdown should inhibit NMD. To test this hypothesis, we silenced expression of SMG-8 and SMG-9 and measured the half lives of PTC-containing $\beta$-globin reporter mRNAs (Fig. 6A). We transfected HeLa TetOff cells with either a wild-type or a PTC-containing $\beta$-globin reporter along with the indicated siRNAs. Cells were treated with doxycycline to repress reporter gene transcription and the half-lives of $\beta$-globin reporter mRNAs were measured. Depletion of SMG-1, SMG-8, or SMG-9 stabilized PTC-containing $\beta$-globin mRNAs (Fig. 6B) but had no effect on the half-life of wild-type mRNAs (Fig. 6C). NS control siRNAs had no effect on either transcript. The half-lives $\left(t_{1 / 2}\right)$ of PTC-mRNAs were estimated to be 140 $\pm 8.9 \mathrm{~min}$ for the NS control, $195.6 \pm 6.9 \mathrm{~min}$ following SMG-8 depletion, $199.5 \pm 7.4 \mathrm{~min}$ following SMG-9 depletion, and $>240$ min following SMG-1 depletion. The half-lives of PTC-containing $\beta$-globin mRNAs are statistically significant compared with the control in the SMG-8 depletion $(P=0.0135)$ and SMG-9 depletion $(P=$ $0.0005)$, respectively. To further confirm the requirements of SMG-8 and SMG-9 for NMD in mammals, we assessed the accumulation of endogenous GAS5 mRNA, a natural target of NMD (Ideue et al. 2007), in SMG-8, SMG-9, or SMG-8/SMG-9 double knockdown cytoplasmic cell extracts. As shown in Figure 6D, the accumulation of GAS5 mRNA was observed by SMG-1 (3.8-fold), SMG-8 (2.9-fold), SMG-9 (2.6-fold), or SMG-8/SMG-9 (threefold) knockdown. All tested proteins were efficiently depleted after siRNA transfection (data not shown).

To exclude the possibility that the suppression of Upf1 phosphorylation and of NMD by SMG-8 knockdown is due to inhibition of translation (Carter et al. 1995), we established a luciferase-based reporter assay to analyze NMD (Supplemental Fig. 11). Knockdown of SMG-8, Upf1, or SMG-6 increased NMD-sensitive luciferase activity, which requires translation (Fig. 6E). No significant effects were observed on the NMD-insensitive luciferase activity (Fig. 6F). All tested proteins were efficiently depleted after siRNA transfection (data not 
Yamashita et al.

A

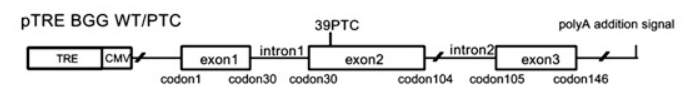

B

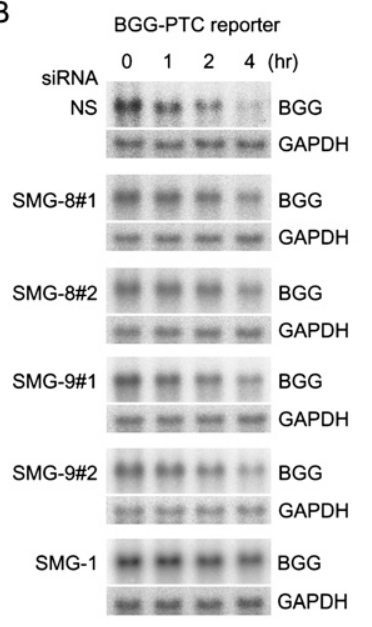

C

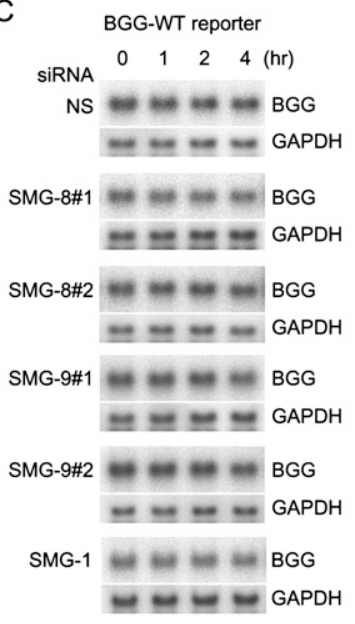

D

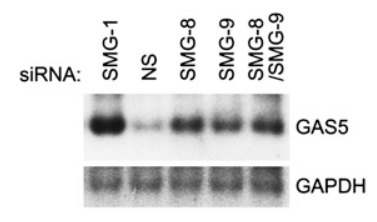

E

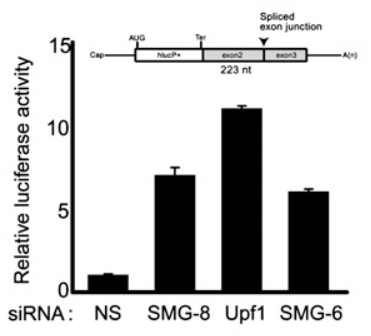

$\mathrm{F}$

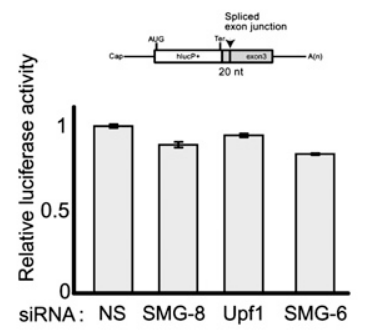

G

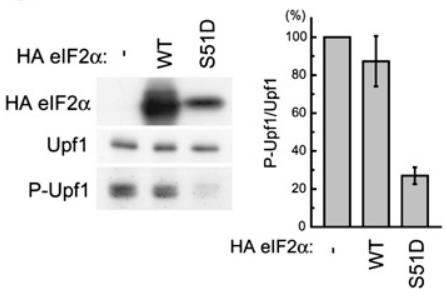

Figure 6. SMG-8 and SMG-9 are required for NMD in mammalian cells. (A) Schematic structure of the $\beta$-globin gene (BGG) wild-type (WT)/PTC reporter plasmid used for the half-life assay. The ORF is represented by boxes and introns and UTRs are represented by lines. $(B-D)$ HeLa TetOff cells were transfected with the siRNAs indicated and simultaneously transfected with Tet-inducible BGG PTC $(B)$ or with BGG wild-type $(C)$ reporter plasmid. After the addition of doxycycline to repress the transcription of reporter plasmids, total RNAs prepared at the times indicated were analyzed by Northern blotting using $\beta$-globin or GAPDH cDNA probes. The quantities of BGG mRNA, normalized to GADPH signals, were plotted (T1/2: half-life). $(B, C)$ Mean values \pm SD from three independent experiments are shown. $(D)$ Forty-eight hours after siRNA transfection, cytoplasmic RNAs were prepared and analyzed by Northern blotting using GAS5 or GAPDH cDNA probes. $(E, F)$ HeLa TetOff cells were transfected with the siRNAs together with plasmids expressing firefly luciferase based NMD reporter $(E)$ or control reporter $(F)$ and renilla luciferase plasmids and subjected to luciferase assay. Relative firefly luciferase activities, which were normalized by renilla luciferase activities, are shown graphically. Mean values \pm SD from three independent experiments are shown. $(G)$ HeLa TetOff cells transfected with the plasmids indicated above. Thirty-three hours after transfection, cells were lysed and analyzed by Western blotting with the indicated antibodies. Mean values \pm SD from three independent experiments are shown.

shown). These results indicate that the inhibition of Upf1 phosphorylation and NMD in SMG-8-depleted cells was not caused by translation suppression.

Although inhibition of translation initiation has been expected to suppress Upf1 phosphorylation, direct evidence was lacking. To evaluate this, we examined the effect of the inhibition of translation initiation on
Upf1 phosphorylation. The over expression of the eIF2 $\alpha$-S51D mutant, which suppresses translation initiation and NMD (Chiu et al. 2004), clearly inhibited Upf1 phosphorylation, whereas wild-type eIF2 $\alpha$ overexpression did not, supporting the above notion (Fig. 6G). These results also indicate that NMD inhibition by SMG-9 depletion was not caused by the 
suppression of translation initiation, since SMG-9 depletion leads to the up-regulation of Upf1 phosphorylation (Fig. 5A).

\section{SMG-8 is required for efficient NMD in C. elegans}

Putative orthologs of SMG-8 and SMG-9 are evident in the genomes of all sequenced metazoa. The C. elegans genes K04B12.3 (smg-8) and Y54E2A.2 (smg-9) are 16.3\% and $14.2 \%$ identical to SMG-8 and SMG-9, respectively (Supplemental Figs. 3, 4). We inhibited expression of $C$. elegans smg-8 and smg-9 by RNAi and investigated in two ways whether the resulting animals exhibited NMD defects. First, we tested $s m g-8(R N A i)$ and $s m g-9(R N A i)$ animals for phenotypic suppression of unc-54(r293). Unc54(r293) is an allele of a muscle myosin heavy chain gene whose mutant mRNA is a substrate of NMD (Hodgkin et al. 1989; Pulak and Anderson 1993). Mutations affecting any of the known smg genes phenotypically suppress the paralysis of unc-54(r293) by eliminating NMD. We performed smg-8(RNAi) and smg-9(RNAi) in both unc-54(r293) single mutants and in unc-54(r293) smg-1(cc546ts) double mutants. Smg-1(cc546ts) is a temperature-sensitive allele that is NMD-defective at $25^{\circ} \mathrm{C}$ but NMD-competent at $20^{\circ} \mathrm{C}$ or below (S. Getz, S. $\mathrm{Zu}$, and A. Fire, pers. comm.). We observe that smg1(cc546ts) mutants grown at permissive temperature $\left(20^{\circ} \mathrm{C}\right.$ or below) are a sensitized genetic background in which weak NMD defects can be detected that are not evident in smg-1(+) strains, presumably because activity

A

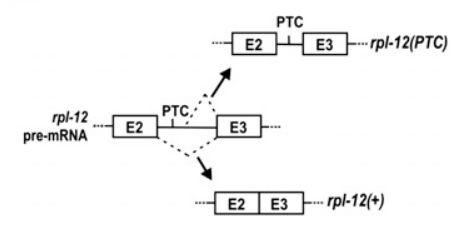

B

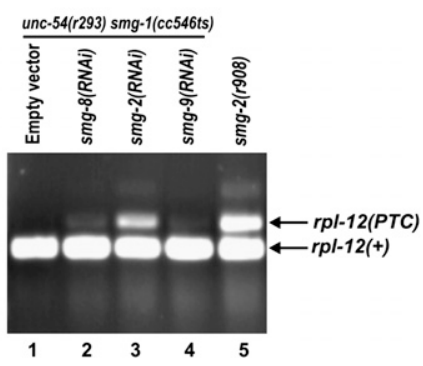

Figure 7. SMG-8 is required for efficient NMD in C. elegans. (A) Alternative splicing of C. elegans rpl-12 pre-mRNAs. Rpl12(PTC) mRNA contains a PTC and is a substrate of NMD. Rpl$12(+)$ does not contain a PTC and is not an NMD substrate. (B) Smg-8(RNAi) weakly inhibits C. elegans NMD. Unc-54(r293) smg-1(cc546ts) animals were fed bacteria expressing the indicated dsRNAs (lanes 2-4) or no dsRNA (lane 1). RNAs were prepared from these strains, converted to cDNA, and amplified with primers that flank the alternatively spliced $r p l-12$ intron. The positions of $r p 1-12(P T C)$ and $r p l-12(+)$ PCR products following gel electrophoresis are indicated. Lane 5 is RT-PCR analysis of the deletion allele $s m g-2(r 908)$ without RNAi treatment. of SMG-1 is compromised or partially inactivated by cc546ts. Smg-8(RNAi) weakly but consistently suppressed the motility defects of unc-54(r293) smg1 (cc546ts) at both $15^{\circ} \mathrm{C}$ and $20^{\circ} \mathrm{C}$ compared with empty vector negative controls. Suppression was weaker than that observed with a smg-2(RNAi) positive control, but the improved motility of unc-54(r293) smg-1(cc546ts); smg-8(RNAi) when compared with unc-54(r293) smg1 (cc546ts) is unambiguous. We observed little or no suppression by $s m g-8(R N A i)$ in $s m g-1(+)$ genetic backgrounds, and suppression by smg-9(RNAi) was not detected in either strain at either temperature.

To confirm that smg-8(RNAi) animals are partially defective for NMD, we performed RT-PCR of rpl-12 mRNA prepared from the animals described above. $R p 1-12$ pre-mRNA is alternatively spliced to yield two mature mRNAs, one of which contains a PTC /see Fig. 7A; Mitrovich and Anderson 2000). Unproductively spliced rpl-12 mRNAs ["rpl-12(PTC)" in Fig. 7A] are substrates of NMD, while productively spliced mRNAs ["rpl-12(+)" in Fig. 7A] are not. The abundance of rpl-12(PTC) PCR products relative to rpl-12(+) products is a measure of NMD efficiency (Mitrovich and Anderson 2000). Smg-8(RNAi) reproducibly stabilized a small fraction of rpl-12(PTC) mRNA in unc-54(r293) smg1(cc546ts) animals (Fig. 7, cf. lanes 2 and 1). The NMD defect of smg-8(RNAi) is weaker that observed in a smg2(RNAi) control (lane 3) and in a smg-2 deletion mutant without RNAi (lane 5). The measured PTC/+ ratios were 0.00 for empty vector, 0.12 for $s m g-8$ (RNAi), 0.53 for smg-2(RNAi), 0.79 for smg-2 deletion mutant, respectively. We did not reliably detect stabilization of rpl12(PTC) mRNA in smg-9(RNAi) animals (lane 4). We conclude from these two tests that $C$. elegans smg8(RNAi) animals are weakly defective for NMD.

\section{Discussion}

SMG-8 and SMG-9 are components of the SMG-1 complex (SMG1C)

Several lines of evidence indicate that SMG-8 and SMG-9 associate tightly with SMG-1 as a complex in vivo. First, immunoprecipitated SMG-1 copurifies with pp130 and pp60 phosphoproteins (Fig. 1A,E; Supplemental Figs. 1, 2), and in vitro phosphorylated pp130 and pp60 are immunoprecipitated by our anti-SMG-8 and -SMG-9 antibodies, respectively (Fig. 1E). Second, pp130 (SMG-8) and pp60 (SMG-9) associate with SMG-1 during anion exchange column chromatography (Supplemental Fig. 1). SMG1C migrates in different chromatographic fractions from other members of PIKKs including ATM, ATR, DNA-PKcs, and mTOR in this condition. This suggests that the SMG1C indigently forms with other PIKKs. Third, SMG-9 immunodepletion demonstrates that the majority of both SMG-1 and SMG-8 form a complex with SMG-9 (Fig. 1F). Fourth, SMG-8 and SMG-9 bind to SMG-1 in both cytoplasmic and nuclear fractions, unlike Upf1 and Upf2 (Supplemental Fig. 6). The significant reduction of SMG-1:SMG-8 binding by depletion of SMG-9 suggests 
that SMG-9 enhances formation of the SMG1C (Figs. 2A, 4A). Consistent with this, we reconstituted a SMG1:SMG-8:SMG-9 ternary complex following overexpression of the proteins only when all three were coexpressed (data not shown). Although we have not yet demonstrated in vivo phosphorylation of SMG-8 and SMG-9, both proteins are phosphorylated by SMG-1 in vitro (Fig. 1, 2; Supplemental Figs. 1, 2). This implies an additional regulatory mechanism of NMD involving the SMG-1 catalytic activity. We additionally observed phosphorylation of SMG-8 and SMG-9 in SMG-1 immunoprecipitates from cell extracts of mouse embryonic fibroblasts TetOff cells, rat $3 \mathrm{Y} 1$ cells, and swine placenta (data not shown). Thus, SMG-8 and SMG-9 phosphorylations are conserved among mammals.

SMG-8 negatively regulates SMG-1 kinase activity in the isolated SMG-1 complex and is also required for the recruitment of SMG-1 to the surveillance complex

Previous studies have established the critical importance of the phosphorylation of Upf1 by SMG-1, leading to the prediction that SMG-1 kinase activity must be suppressed until SURF associates with EJC. We provided biochemical evidence that SMG-8 suppresses the kinase activity of SMG-1 in the isolated SMG-1 complex (Fig. 2). Importantly, SMG-8 has an additional role in recruitment of SMG-1 to the EJC, an essential step for activation of SMG-1 kinase (Fig. 4B,C). Consistently, SMG-8 were present in most SMG1Cs, including the SURF complex (Figs. 1F, 3A). Thus, SMG-8 clearly has dual activity, suppression of the kinase activity and the recruitment of the SMG1C to the EJC. This dual regulatory role of SMG-8 on the kinase activity of SMG-1 can explain the mechanism how SMG-1 kinase is suppressed until SURF associates with EJC on post-spliced mRNAs. A requirement for SMG-8 to recruit SMG-1 to the EJC insures that catalytically inactive SMG-1 is delivered to the surveillance complex. Upf1 phosphorylation then occurs only after EJC recognition and after remodeling of the mRNP complex. Consistent with this, SMG-8 is required for Upf1 phosphorylation in vivo (Fig. 5; Supplemental Fig. 10). Accumulation of a Upf1:SMG-1 complex in the absence of SMG-8 suggests that the Upf1:SMG1C complex forms before the SURF complex formation (Fig. 4A; Supplemental Fig. 9). Thus, SMG-8 likely recruits the Upf1:SMG1C complex to a ribosome stalled at PTCs (see Fig. 8).

While Upf1 can be an efficient substrate of PIKKs, namely SMG-1, ATM, ATR, and DNA-PKcs, in various situations, SMG-1 is likely the only PIKK involved in the phosphorylation of Upf1 during NMD (Yamashita et al. 2001; Brumbaugh et al. 2004; Azzalin and Lingner 2006; Muller et al. 2007). The presence of SMG-1 specific binding partner, SMG-8, may guarantee the proper substratekinase association between the SMG1C and Upf1, and correct recruitment of Upf1:SMG1C to the mRNA surveillance complex. The direct binding of Upf2 to the C-terminal half of SMG-1 (Kashima et al. 2006) might activate SMG-1 to phosphorylate Upf1 in the mRNA

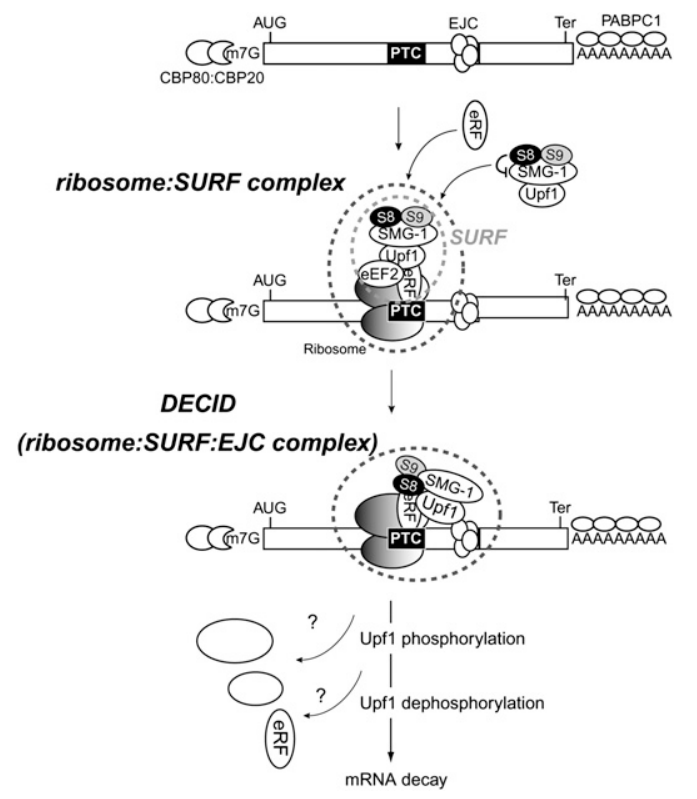

Figure 8. A model for the process from nonsense termination to mRNA decay. SMG1C, the SMG-1:SMG-8:SMG-9 complex, binds Upf1 before SURF formation. Then, the SMG1C:Upf1 complex interacts with eRF to form SURF, which associates with the ribosome and eEF2 on mRNPs containing the CBP20:CBP80 complex and PABPC1 after or during recognition of translation termination codons. SMG-8 is likely to mediate the recruitment of SMG-1 to the ribosome:SURF complex and to suppress SMG-1 kinase activity until the ribosome:SURF complex locates the EJC. SMG-9 is required for the efficient association between SMG-1 and SMG-8. The ribosome:SURF complex is most likely the translation termination complex operating during NMD, and looks for a downstream EJC to form the DECID (the ribosome:SURF:EJC complex). DECID formation likely induces SMG-1-dependent Upf1 phosphorylation, which leads to the recruitment of phospho-Upf1-specific binding proteins, such as SMG-5 and SMG-7 and the dissociation of the ribosome and eRF from Upf1 to advance mRNA decay. (AUG) Start codon; (Ter) termination codon; (PTC) premature termination codon; (EJC) exon junction complex; (eRF) eRF1: eRF3 complex; (S8) SMG-8; (S9) SMG-9; (P) phosphate group; (SURF) SMG-1:Upf1:eRF1:eRF3 complex; (DECID) decay-inducing complex.

surveillance complex, since other PIKK member kinases are also activated by the modification or binding of activator proteins to their PIKK regulatory domain (Mordes et al. 2008).

In contrast to depletion of SMG-8, depletion of SMG-9 showed no significant changes of proteins associated with Y14 and Upf1 (Fig. 4C,D). These results suggest that SMG-9 depletion does not cause the SURF or the ribosome:Upf1:eRF1:eRF3:EJC complex to accumulate (see below). This implies that SMG-8 can recruit the Upf1:SMG-1 complex to ribosomes stalled at PTCs in the absence of SMG-9, even though SMG-9 is involved in SMG-1:SMG-8 interactions (Figs. 2A, 4A). Depletion of SMG-9 causes increased accumulation of phosphorylated Upf1, suggesting a specific role of SMG-9 in regulation of the Upf1 
phosphorylation cycle (Fig. 5A). The reduced interaction between SMG-1 and the EJC in SMG-9-depleted cells suggests the possibility that SMG-9 depletion affects recruitment and/or activation of the Upf1 dephosphorylation components. Specific association of SMG-7 with the N-terminal region of SMG-1 supports this possibility (Kashima et al. 2006; Morita et al. 2007). Further analysis is needed to clarify the role of SMG-9 in cycles of Upf1 phosphorylation and dephosphorylation in vivo.

\section{The PTC recognition complex during NMD}

We showed that the SURF complex associates with ribosomes on mRNPs prior to phosphorylation of Upf1, which is a critical step in NMD. Several lines of evidence support this conclusion. First, immunoprecipitated Upf1C126S, which is a poorly phosphorylated mutant of Upf1 that accumulates the SURF complex (Fig. 3B; Kashima et al. 2006), copurifies with increased amounts of ribosomal proteins rpL11, rpL7a, rpS3, and rpS6 compared with the immunoprecipitation of wild-type Upf1 in the presence of cycloheximide (Fig. 3B). Second, the Upf1 complex isolated from Y14-depleted cell extracts, which are also conditions that accumulate the SURF complex (Kashima et al. 2006), contains increased amounts of ribosomal proteins (Fig. 3C). Third, immunoprecipitation of Upf1 from Y14-depleted cell extracts copurify with significantly increased amounts of PABPC1, CBP20, and CBP80, but only in the absence of Micrococcal nuclease treatment (Fig. 3D). These results all indicate an important association between the SURF:ribosome and mRNPs. Because the SURF complex associates with both $60 \mathrm{~S}$ and $40 \mathrm{~S}$ ribosomal subunit proteins (Fig. 3B,C), and because the $60 \mathrm{~S}$ ribosome is released from mRNA together with (Pisarev et al. 2007) or after (Demeshkina et al. 2007) dissociation of release factors from mRNAs, the ribosome associated with the SURF complex is most likely the $80 \mathrm{~S}$ ribosome, which presumably is stalled at PTCs together with release factors (Fig. 3E).

In addition to defining a ribosome:SURF complex on mRNPs, we found the existence of the physical interaction between PTC-stalled ribosome and exon junction via protein-protein interactions. The following observations strongly support this idea. First, Y14 immunoprecipitated from SMG-8-depleted cell extracts copurifies with increased amounts of the ribosome:SURF complex components Upf1, eRF1, eRF3, and 60S and 40S ribosomal proteins (Fig. 4C). Second, SMG-8 or SMG-1 depletion causes an enhancement of the association between Upf1 and EJC components, 60S and 40S ribosomal proteins in the presence of RNase (Fig. 4D). Importantly, the ribosome:Upf1:eRF1:eRF3:EJC complex observed following SMG-8 depletion appeared on mRNPs, which we demonstrated by the RNase-sensitive association of Upf1 with PABPC1 and CBP80 (Fig. 4E). The presence of both $60 \mathrm{~S}$ and $40 \mathrm{~S}$ ribosomal proteins and release factors in the ribosome:Upf1:eRF1:eRF3:EJC:mRNP complex suggests that intact $80 \mathrm{~S}$ ribosomes are stalled at PTCs (Fig. 4F). Taken together with the essential role of the SMG-1 kinase activity for dissociation of eRF3 from Upf1 (Kashima et al. 2006), these results suggest that the ribosome and release factors are not able to dissociate from the Upf1:EJC complex without Upf1 phosphorylation. Thus, SMG-8-mediated SMG1C recruitment to the EJC is required for subsequent Upf1 phosphorylation by SMG-1 and these processes are essential to proceed the remodeling of mRNA surveillance complex. It is worth noting that both SMG-1 (Kashima et al. 2006) and Upf1 (Fig. 4C,D) can bind to EJC without each other and both can bind directly to Upf2 (Kashima et al. 2006; Chamieh et al. 2008), although our results indicate the SMG-8dependent recruitment of the Upf1:SMG1C complex to the EJC (Fig. 4A; Supplemental Fig. 9). From these results, we propose that two independent protein-protein interactions, Upf1:EJC and SMG1C:EJC, are required for the formation of the ribosome:SURF:EJC complex, DECID, and the discrimination of termination codons as premature (our model is depicted in Fig. 8). Further analysis is required to clarify our model, such as in vitro reconstitution, since immunoprecipitation analysis could not exclude the association and/or dissociation of protein complex during cell lysis.

Our study of mRNP remodeling associated with NMD provides insights into conserved mechanisms of both premature and normal translation termination in vivo. The involvement of eRF1 and eRF3 in both types of termination implies the fundamental importance of the eRF1:eRF3 complex. The presence of eEF2 in the ribosome:SURF complex (Fig. 3B,C) might also suggest mechanistic similarity of both types of termination, because eEF2 is proposed to be involved in normal termination (Demeshkina et al. 2007). Since the accumulation of eEF2, eRF1, and eRF3 is not significant as that of ribosomal proteins (Fig. 3B,C), the association among Upf1 and them might have an additional role for Upf1dependent post-transcriptional regulation (Kaygun and Marzluff 2005; Kim et al. 2005).

Although premature and normal translation termination are similar, clear differences exist between the two types of termination events. For example, we did not detect PABPC1, a component of the normal translation termination complex (Uchida et al. 2002), in either the ribosome:SURF complex or the DECID complex in the presence of RNase, even though it accumulates in these mRNP complexs (Figs. 3D, 4E). We also did not detect the CBP20:CBP80 complex in the ribosome:SURF complex or the DECID complex after micrococcal nuclease treatment (Figs. 3D, 4E), in contrast to previous observations (Hosoda et al. 2005). These results suggest that fundamental differences exist between premature and normal translation termination. The premature translation termination complex, namely, the ribosome:SURF complex and the DECID complex, does not appear to communicate with the $5^{\prime}$ cap or the poly(A) tail, whereas the normal translation termination complex communicates with both of these elements via protein-protein interactions to improve both efficient translation termination (Cosson et al. 2002; Ivanov et al. 2008) and translation initiation (Uchida et al. 2002). 


\section{SMG-8 and SMG-9 are involved in NMD}

The following observations indicate that SMG-8 and SMG-9 are involved in NMD. First, depletion of either SMG-8 or SMG-9 in mammalian cells led to inhibition of NMD (Fig. 6). Second, treatment of a sensitized $C$. elegans strain with $s m g-8$ dsRNA produced a weak Smg phenotype as judged both by phenotypic suppression of unc-54(r293) and by increased abundance of rpl-12(PTC) mRNA (Fig. 7). The evolutionary conservation of SMG-8 and SMG-9 among all eukaryotes that have SMG-1 in their genome implies that the conserved PTC recognition complex exists in a similar manner to that we describe for the mammalian DECID complex, even though neither Drosophila melanogaster nor C. elegans requires splicing and the EJC for NMD (Pulak and Anderson 1993; Gatfield et al. 2003; Longman et al. 2007). Consistent with this view, SMG-2, the C. elegans ortholog of Upf1, strongly marks PTC-containing mRNAs both in wild-type and in a smg-1 deletion mutant (Johns et al. 2007). Marking of $C$. elegans mRNAs by SMG-2 depends on SMG-3 and SMG-4 (Upf2 and Upf3 orthologs) (Johns et al. 2007), which further supports existence of the evolutionarily conserved PTC recognition complex described in this study.

In conclusion, we identified two novel subunits of the SMG-1 complex, SMG-8 and SMG-9, that act as regulators of NMD. We identified a complex involving the ribosome, Upf1, SMG-1, eRF1, eRF3, and the EJC that bridges the ribosome and the EJC on mRNPs, and this complex formation is regulated by SMG- 8 . The complex we describe is the first direct demonstration of a physical interaction between the ribosome and the exon-exon junction via protein-protein interactions. Together with identification of the ribosome:SURF complex on mRNPs, further analyses of the sequential remodeling of the surveillance complex by the inactivation of NMD factors will clarify the details of the events from translation termination to mRNA decay in vivo. Finally, we revealed the biochemical nature of the SMG1C with two regulatory subunits, SMG-8 and SMG-9. In addition to NMD, SMG-1 is involved in genome surveillance, telomere maintenance, the suppression of TNF $\alpha$-induced apoptosis and hyperoxia-induced p53 activation (Brumbaugh et al. 2004; Chen et al. 2006; Azzalin et al. 2007; Gehen et al. 2008; Oliveira et al. 2008). Further analysis of the roles of the SMG1C will accelerate the understanding of the mechanism underlying the responses of cells against various stresses.

\section{Materials and methods}

\section{Plasmids and antibodies and siRNAs}

The expression vector for CBP-SMG-8r (2-991), SR-strep-HAeIF2 $\alpha$ wild type and SR-strep-HA-eIF2 $\alpha$-S51A were constructed in the mammalian expression vector pNTAP (Stratagene) or pSRstrep-HA, following standard procedures. CBP-SMG-8r was mutated at nucleotides 198 and 201 of coding sequence for siRNA SMG-8\#1 resistance by site-directed mutagenesis. Flag-SMG-1, HA-Upf1 wild type, HA-Upf1-C126S, pTRE-BGG wild type, and pTRE-BGG PTC reporter plasmids were constructed as described previously (Yamashita et al. 2001; Ohnishi et al. 2003; Kashima et al. 2006).

Anti-SMG-1-IP, which used in Figure 4B, anti-SMG-8, and anti-SMG-9 antibodies were generated against recombinant human SMG-1 (amino acids 1827-1926), SMG-8 (amino acids 563-771), or SMG-9 (amino acids 21-75) proteins fused to GST. Affinity purification of the antibodies was performed following standard procedures. Mouse monoclonal antibodies raised against Upf1 (5C3) and phospho (P)-Upf1 (8E6, 7D5, 7H1, 9C2, and 2C9) were generated with a standard method using KLH conjugated peptide antigens: EEDEEDTYYTKDLPIHAC for Upf1 or LSQPELS[-P]QDSYLG for P-Upf1 (Supplemental Figs. 7, 12). Antibodies to SMG-1, Upf1, Upf2, Upf3a, Upf3b, SMG-7, and eIF4A3 (clone 3F1) have been described previously (Yamashita et al. 2001; Ohnishi et al. 2003; Kashima et al. 2006). Antibodies to Magoh (Abcam), Y14 (Sigma), eIF4A3 (ProteinTech), eRF1 (Abcam), eRF3 (Abcam), rpL11 (Invitrogen), rpL7a (Cell Signaling Technology), rpS6 (Cell Signaling Technology), rpS16 (Abcam), rpS3 (Cell Signaling Technology), eEF2 (Cell Signaling Technology), PABPC1 (Abcam), CBP20 (Abgent), CBP80 (ProteinTech), $\beta$-actin (Sigma), and GAPDH (Abcam) were used. The antitag antibodies (HA, Flag) were obtained commercially. The following target sequences were used: SMG-8\#1, siGENOME duplex D-016480-02 (Dharmacon); SMG-8\#2, siGENOME duplex D-016480-03 (Dharmacon); SMG-9\#1, siGENOME duplex D-020561-01 (Dharmacon); SMG-9\#2, siGENOME duplex D020561-04 (Dharmacon); SMG-1, AAGTGTATGTGCGCCAAA GTA; Upf1, AAGATGCAGTTCCGCTCCATT; Y14, FAACGCT CTGTTGAAGGCTGGA， AATTCGCAGAATATGGGGAAA; SMG-6, AAGCCAGTGATACAGCGAATT; NS, AATTCTCCG AACGTGTCACGT.

\section{Transfection with plasmids and siRNAs}

HeLa TetOff cells (Clontech) were grown in Dulbecco's modified Eagle's medium (DMEM) supplemented with $10 \%$ fetal bovine serum, $100 \mathrm{U} / \mathrm{mL}$ penicillin, and $100 \mu \mathrm{g} / \mathrm{mL}$ streptomycin. Plasmid transfections were performed in six-well plates, $10-\mathrm{cm}$ dishes, or 15-cm dishes using Polyfect (Qiagen), Lipofectamin 2000 (Invitrogen), or Lipofectamine LTX (Invitrogen) according to the manufacturer's recommendations. For the immunoprecipitation of HA- or Flag-tagged proteins, transfected cells were harvested $36 \mathrm{~h}$ after transfection. siRNA transfections were performed in 12-well plates or in 15-cm dishes using RNAifect (Qiagen) or Hiperfect (Qiagen) according to the manufacturer's protocol, and cells were harvested 48-72 h later.

\section{Immunoprecipitation and Western blot analysis}

HeLa TetOff cells were transfected using Lipofectamin 2000 (Invitrogen), Lipofectamine LTX (Invitrogen), Polyfect (Qiagen), RNAifect (Qiagen), or Hiperfect (Qiagen), and lysed in T-buffer (20 mM HEPES-NaOH at pH 7.5, $50 \mathrm{mM} \mathrm{NaCl}, 0.05 \%$ Tween 20, $2.5 \mathrm{mM} \mathrm{MgCl}, 0.5 \mathrm{mM}$ DTT, $100 \mathrm{nM}$ okadaic acid [Calbiochem], protease inhibitor cocktail [Roche], phosphatase inhibitor cocktail [Roche], $100 \mu \mathrm{g} / \mathrm{mL}$ cycloheximide [Sigma], $100 \mu \mathrm{g} / \mathrm{mL}$ RNaseA [Qiagen]) or in NP40-buffer (50 mM Tris- $\mathrm{HCl}$ at $\mathrm{pH} 7.4$, $150 \mathrm{mM} \mathrm{NaCl}, 0.4 \%$ NP40, $2 \mathrm{mM} \mathrm{MgCl}_{2}, 1 \mathrm{mM}$ DTT, $100 \mathrm{nM}$ okadaic acid [Calbiochem], protease inhibitor cocktail [Roche], phosphatase inhibitor cocktail [Roche], $100 \mu \mathrm{g} / \mathrm{mL}$ cycloheximide [Sigma], $100 \mu \mathrm{g} / \mathrm{mL}$ RNaseA [Qiagen]). The soluble fractions were subjected to preclear and then incubated with antibodies preadsorbed on $30 \mu \mathrm{L}$ of protein $\mathrm{G}$ or protein $\mathrm{A}$ Sepharose (GE Biotech) for $2 \mathrm{~h}$ at $4^{\circ} \mathrm{C}$ with rotation. To immunoprecipitate HA-Upf1, $25 \mu \mathrm{L}$ of anti-HA affinity matrix (Roche) was used. After washing with RNase(-) lysis buffer, 
the immunocomplexes were boiled in SDS sample buffer, and then analyzed by Western blotting. In experiments where antiHA affinity matrix or Upf1-monoclonal antibody was used, proteins recovered on the matrix were eluted by incubation for $15 \mathrm{~min}$ at $37^{\circ} \mathrm{C}$ with RNase(-) lysis buffer containing $1 \mathrm{mg} / \mathrm{mL}$ HA-peptide or $1 \mathrm{mg} / \mathrm{mL}$ Upfl peptide, boiled with SDS sample buffer, and analyzed by Western blotting. For micrococcal nuclease treatment, the immunoprecipitates were washed with $1 \times$ micrococcal nuclease buffer (Takara) and incubated with $3000 \mathrm{U} / \mathrm{mL}$ micrococcal nuclease (Takara) in $300 \mu \mathrm{L}$ of $1 \times$ micrococcal nuclease buffer containing phosphatase inhibitor cocktail (Sigma), protease inhibitor cocktail (Roche), RNasin (Promega), and $100 \mu \mathrm{g} / \mathrm{mL}$ cycloheximide for $30 \mathrm{~min}$ at $25^{\circ} \mathrm{C}$ and immunocomplexes were then washed with RNase(-) wash buffer. All proteins in Western blot experiments were detected with the ECL Western blot detection kit (GE Biotech) or Lumi Light (Roche), and quantified with a LuminoImager, LAS-3000, and Science Lab 2001 Image Gauge software (Fuji Photo Film). All experiments were performed at least three times, and typical results are shown.

\section{In vitro kinase assay}

Cells were lysed in F-buffer (20 mM Tris- $\mathrm{HCl}$ at $\mathrm{pH} 7.5,0.25 \mathrm{M}$ sucrose, $1.2 \mathrm{mM}$ EGTA, $20 \mathrm{mM} \beta$-mercaptoethanol, $1 \%$ Triton X-100, 0.5\% Nonidet P-40, $150 \mathrm{mM} \mathrm{NaCl}$, phosphatase inhibitors, protease inhibitors). Endogenous SMG-1 was immunoprecipitated using anti-SMG-1-C antiserum (Yamashita et al. 2001). Immunoprecipitates were washed with F-buffer containing 0.25 $\mathrm{M} \mathrm{LiCl}$ and $1 \times$ kinase reaction buffer $(10 \mathrm{mM}$ HEPES-KOH at $\mathrm{pH}$ 7.5, $50 \mathrm{mM} \mathrm{NaCl}, 10 \%$ [w/v] glycerol, $10 \mathrm{mM} \mathrm{MnCl}_{2}, 50 \mathrm{mM}$ $\beta$-glycerophosphate, $1 \mathrm{mM}$ DTT, $100 \mathrm{nM}$ okadaic acid, protease inhibitor cocktail [Sigma]). Kinase reactions were carried out in $50 \mu \mathrm{L}$ of $1 \times$ kinase reaction buffer containing $50 \mu \mathrm{M}$ ATP and 5 $\mu \mathrm{Ci}\left[\gamma^{-32} \mathrm{P}\right]$ ATP for $20 \mathrm{~min}$ at $30^{\circ} \mathrm{C}$ or the indicated times with or without the indicated substrates. After separation by SDS-PAGE, phosphoproteins were visualized by autoradiography. The extent of phosphorylation of the proteins was evaluated using an imaging analyzer (BAS2500, FujiFilm).

\section{NMD analysis in mammalian cells}

NMD analysis was performed in essentially the same manner as described elsewhere (Yamashita et al. 2001; Ohnishi et al. 2003), except that HeLa TetOff cells $\left(1.8 \times 10^{6}\right)$ were transfected with siRNAs, which are indicated in Figure 6, B and C, in combination with $1.5 \mu \mathrm{g}$ of pTRE-BGG wild type or PTC. Briefly, $60 \mathrm{~h}$ after transfection, doxycycline was added at time zero to inhibit de novo transcription, and total RNAs isolated at the indicated time points were analyzed by Northern blotting using $\beta$-globin or GAPDH (control) cDNA probes. The quantities of BGG mRNA, normalized to GADPH signals, are graphed in Figure 6, B and C. Values in Figure 6, B, E, and F, represent the mean \pm SD for three to four independent experiments. The half-lives of PTCcontaining $\beta$-globin from NS, SMG-8 (\#1 and \#2) or SMG-9 (\#1 and \#2) siRNA-treated extracts were calculated from three (NS) or six (SMG-8 and SMG-9) independent experiment. Mean values \pm SE were shown in the Results section. The two-tailed Student's $t$-test (Microsoft Excel 2003) was used to analyze the differences between the pairs of groups. Values were regarded significant at $P<0.05$. Data in Figure 6C is the average of two independent experiments. For the GAS5 mRNA accumulation assay, HeLa TetOff cells were transiently transfected with siRNAs indicated in Figure 6D. After 48 h of culture, cells were harvested and cytoplasmic RNAs were isolated. Northern blotting was performed using full length GAS5 or GAPDH cDNA probes. The quantities of GAS5 mRNA, normalized to GAPDH mRNA signals, are written in the Results section. Two independent experiments were performed, and the typical result is shown in Figure 6D. The luciferase-based NMD assay system is described in the Supplemental Material.

\section{RNAi, motility analysis, and RT-PCR in C. elegans}

For RNAi, the HT115(DE3) strain transfected with target plasmids or negative control (vehicle L4440 or pBlueScriptII) was cultured in LB medium containing $25 \mu \mathrm{g} / \mathrm{mL}$ carbenicillin (Sigma) and $12.5 \mu \mathrm{g} / \mathrm{mL}$ tetracycline (Sigma) overnight. Eighty microliters of liquid culture were spread on NGM agar containing $1 \mathrm{mM}$ IPTG (Sigma) and $25 \mu \mathrm{g} / \mathrm{mL}$ carbenicillin and incubated overnight at $30^{\circ} \mathrm{C}$ to induce T7 RNA polymerase and allow dsRNA synthesis. unc-54(r293), RNAi high-sensitive and smg-1 temperature-sensitive strain (genotype unc-54[r293]/smg1[cc546ts]/rrf-3[pk1426]), and unc-54(r293) strain (genotype unc54[r293]) were used as test C. elegans and maintained with E. coli strain OP50 grown on NGM agar as standard method. L4 larvaeyoung adult worms were transferred on HT115(DE3) lawn and incubated for $24 \mathrm{~h}$ at $15^{\circ} \mathrm{C}$. Mortality recovery phenotypes were observed for $24 \mathrm{~h}$.

For RT-PCR, total RNA was extracted from mixed-stage animals using TRIzol (Invitrogen). First-strand synthesis was performed using random hexamers and SuperScript III RT (Invitrogen). Rpl-12 mRNA was amplified using primers on either side of the alternatively spliced intron (Johns et al. 2007). Products were separated on $1.5 \%$ ethidium bromide-stained agarose gels.

\section{Acknowledgments}

We thank Dr. G. Dreyfuss (Howard Hughes Medical Institute and Department of Biochemistry and Biophysics, University of Pennsylvania School of Medicine) for the eIF4A3 antibody. We thank Dr. A. Grimson and W. Wang for initial work on C. elegans RNAi. We also thank Y. Kikuchi for technical support. This work was supported, in part, by grants from the Japan Society for the Promotion of Science (to A.Y., S.O. I.K., and N.I.), from the Ministry of Education, Culture, Sports, Science and Technology of Japan (to S.O.), from the Japan Science and Technology Corporation (to A.Y. and S.O.), from the Mitsubishi Foundation (to S.O.), and from the Yokohama Foundation for Advancement of Medical Science. I.K. was a Research Fellow of the Japan Society for the Promotion of Sciences and is currently a Research Fellow of the Max Planck Institute. N.I. is a Research Fellow of the Japan Society for the Promotion of Sciences.

\section{References}

Amrani, N., Sachs, M.S., and Jacobson, A. 2006. Early nonsense: mRNA decay solves a translational problem. Nat. Rev. Mol. Cell Biol. 7: 415-425.

Azzalin, C.M. and Lingner, J. 2006. The human RNA surveillance factor UPF1 is required for $S$ phase progression and genome stability. Curr. Biol. 16: 433-439.

Azzalin, C.M., Reichenbach, P., Khoriauli, L., Giulotto, E., and Lingner, J. 2007. Telomeric repeat containing RNA and RNA surveillance factors at mammalian chromosome ends. Science 318: 798-801.

Baliga, B.S., Pronczuk, A.W., and Munro, H.N. 1969. Mechanism of cycloheximide inhibition of protein synthesis in a cell-free system prepared from rat liver. J. Biol. Chem. 244: 44804489. 
Belgrader, P., Cheng, J., and Maquat, L.E. 1993. Evidence to implicate translation by ribosomes in the mechanism by which nonsense codons reduce the nuclear level of human triosephosphate isomerase mRNA. Proc. Natl. Acad. Sci. 90: 482-486.

Brumbaugh, K.M., Otterness, D.M., Geisen, C., Oliveira, V., Brognard, J., Li, X., Lejeune, F., Tibbetts, R.S., Maquat, L.E., and Abraham, R.T. 2004. The mRNA surveillance protein hSMG-1 functions in genotoxic stress response pathways in mammalian cells. Mol. Cell 14: 585-598.

Cali, B.M. and Anderson, P. 1998. mRNA surveillance mitigates genetic dominance in Caenorhabditis elegans. Mol. Gen. Genet. 260: 176-184.

Carter, M.S., Doskow, J., Morris, P., Li, S., Nhim, R.P., Sandstedt, S., and Wilkinson, M.F. 1995. A regulatory mechanism that detects premature nonsense codons in T-cell receptor transcripts in vivo is reversed by protein synthesis inhibitors in vitro. J. Biol. Chem. 270: 28995-29003.

Chamieh, H., Ballut, L., Bonneau, F., and Le Hir, H. 2008. NMD factors UPF2 and UPF3 bridge UPF1 to the exon junction complex and stimulate its RNA helicase activity. Nat. Struct. Mol. Biol. 15: 85-93.

Chang, Y.F., Imam, J.S., and Wilkinson, M.F. 2007. The nonsensemediated decay RNA surveillance pathway. Annu. Rev. Biochem. 76: 51-74.

Chen, Z., Robin, C., Damiano, J., Lydall, J., Lumb, C., Smith, K., Blasetti, A., Daborn, P.J., Heckel, D., McKenzie, J.A., et al. 2006. Positional cloning of a cyromazine resistance gene in Drosophila melanogaster. Insect Mol. Biol. 15: 181-186.

Chiu, S.Y., Lejeune, F., Ranganathan, A.C., and Maquat, L.E. 2004. The pioneer translation initiation complex is functionally distinct from but structurally overlaps with the steadystate translation initiation complex. Genes \& Dev. 18: 745754.

Conti, E. and Izaurralde, E. 2005. Nonsense-mediated mRNA decay: Molecular insights and mechanistic variations across species. Curr. Opin. Cell Biol. 17: 316-325.

Cosson, B., Couturier, A., Chabelskaya, S., Kiktev, D., IngeVechtomov, S., Philippe, M., and Zhouravleva, G. 2002. Poly(A)-binding protein acts in translation termination via eukaryotic release factor 3 interaction and does not influence [PSI(+)] propagation. Mol. Cell. Biol. 22: 3301-3315.

Demeshkina, N., Hirokawa, G., Kaji, A., and Kaji, H. 2007. Novel activity of eukaryotic translocase, eEF2: Dissociation of the $80 \mathrm{~S}$ ribosome into subunits with ATP but not with GTP. Nucleic Acids Res. 35: 4597-4607.

Gatfield, D., Unterholzner, L., Ciccarelli, F.D., Bork, P., and Izaurralde, E. 2003. Nonsense-mediated mRNA decay in Drosophila: At the intersection of the yeast and mammalian pathways. EMBO J. 22: 3960-3970.

Gehen, S.C., Staversky, R.J., Bambara, R.A., Keng, P.C., and O'Reilly, M.A. 2008. hSMG-1 and ATM sequentially and independently regulate the G1 checkpoint during oxidative stress. Oncogene 27: 4065-4074.

Grimson, A., O'Connor, S., Newman, C.L., and Anderson, P. 2004. SMG-1 is a phosphatidylinositol kinase-related protein kinase required for nonsense-mediated mRNA Decay in Caenorhabditis elegans. Mol. Cell. Biol. 24: 7483-7490.

Hodgkin, J., Papp, A., Pulak, R., Ambros, V., and Anderson, P. 1989. A new kind of informational suppression in the nematode Caenorhabditis elegans. Genetics 123: 301-313.

Holbrook, J.A., Neu-Yilik, G., Hentze, M.W., and Kulozik, A.E. 2004. Nonsense-mediated decay approaches the clinic. Nat. Genet. 36: 801-808.

Hosoda, N., Kim, Y.K., Lejeune, F., and Maquat, L.E. 2005. CBP80 promotes interaction of Upf1 with Upf2 during nonsense-mediated mRNA decay in mammalian cells. Nat. Struct. Mol. Biol. 12: 893-901.

Ideue, T., Sasaki, Y.T., Hagiwara, M., and Hirose, T. 2007. Introns play an essential role in splicing-dependent formation of the exon junction complex. Genes \& Dev. 21: 19931998.

Ivanov, P.V., Gehring, N.H., Kunz, J.B., Hentze, M.W., and Kulozik, A.E. 2008. Interactions between UPF1, eRFs, PABP and the exon junction complex suggest an integrated model for mammalian NMD pathways. EMBO J. 27: 736-747.

Jaillon, O., Bouhouche, K., Gout, J.F., Aury, J.M., Noel, B., Saudemont, B., Nowacki, M., Serrano, V., Porcel, B.M., Segurens, B., et al. 2008. Translational control of intron splicing in eukaryotes. Nature 451: 359-362.

Johns, L., Grimson, A., Kuchma, S.L., Newman, C.L., and Anderson, P. 2007. Caenorhabditis elegans SMG-2 selectively marks mRNAs containing premature translation termination codons. Mol. Cell. Biol. 27: 5630-5638.

Kashima, I., Yamashita, A., Izumi, N., Kataoka, N., Morishita, R., Hoshino, S., Ohno, M., Dreyfuss, G., and Ohno, S. 2006. Binding of a novel SMG-1-Upf1-eRF1-eRF3 complex (SURF) to the exon junction complex triggers Upf1 phosphorylation and nonsense-mediated mRNA decay. Genes \& Dev. 20: 355367.

Kaygun, H. and Marzluff, W.F. 2005. Regulated degradation of replication-dependent histone mRNAs requires both ATR and Upf1. Nat. Struct. Mol. Biol. 12: 794-800.

Kim, Y.K., Furic, L., Desgroseillers, L., and Maquat, L.E. 2005. Mammalian Staufenl recruits Upfl to specific mRNA 3'UTRs so as to elicit mRNA decay. Cell 120: 195-208.

Kuzmiak, H.A. and Maquat, L.E. 2006. Applying nonsensemediated mRNA decay research to the clinic: Progress and challenges. Trends Mol. Med. 12: 306-316.

Longman, D., Plasterk, R.H., Johnstone, I.L., and Caceres, J.F. 2007. Mechanistic insights and identification of two novel factors in the C. elegans NMD pathway. Genes \& Dev. 21: 1075-1085.

Mitrovich, Q.M. and Anderson, P. 2000. Unproductively spliced ribosomal protein mRNAs are natural targets of mRNA surveillance in C. elegans. Genes \& Dev. 14: 2173-2184.

Mordes, D.A., Glick, G.G., Zhao, R., and Cortez, D. 2008. TopBP1 activates ATR through ATRIP and a PIKK regulatory domain. Genes \& Dev. 22: 1478-1489.

Morita, T., Yamashita, A., Kashima, I., Ogata, K., Ishiura, S., and Ohno, S. 2007. Distant $\mathrm{N}$ - and C-terminal domains are required for intrinsic kinase activity of SMG-1, a critical component of nonsense-mediated mRNA decay. J. Biol. Chem. 282: 7799-7808.

Muller, B., Blackburn, J., Feijoo, C., Zhao, X., and Smythe, C. 2007. DNA-activated protein kinase functions in a newly observed S phase checkpoint that links histone mRNA abundance with DNA replication. J. Cell Biol. 179: 13851398.

Ohnishi, T., Yamashita, A., Kashima, I., Schell, T., Anders, K.R., Grimson, A., Hachiya, T., Hentze, M.W., Anderson, P., and Ohno, S. 2003. Phosphorylation of hUPF1 induces formation of mRNA surveillance complexes containing hSMG-5 and hSMG-7. Mol. Cell 12: 1187-1200.

Oliveira, V., Romanow, W.J., Geisen, C., Otterness, D.M., Mercurio, F., Wang, H.G., Dalton, W.S., and Abraham, R.T. 2008. A protective role for the human SMG-1 kinase against tumor necrosis factor- $\alpha$-induced apoptosis. J. Biol. Chem. 283: $13174-13184$

Pisarev, A.V., Hellen, C.U., and Pestova, T.V. 2007. Recycling of eukaryotic posttermination ribosomal complexes. Cell 131: 286-299. 
Pulak, R. and Anderson, P. 1993. mRNA surveillance by the Caenorhabditis elegans smg genes. Genes \& Dev. 7: 18851897.

Shiloh, Y. 2003. ATM and related protein kinases: Safeguarding genome integrity. Nat. Rev. Cancer 3: 155-168.

Uchida, N., Hoshino, S., Imataka, H., Sonenberg, N., and Katada, T. 2002. A novel role of the mammalian GSPT/eRF3 associating with poly(A)-binding protein in Cap/Poly(A)-dependent translation. J. Biol. Chem. 277: 50286-50292.

Usuki, F., Yamashita, A., Higuchi, I., Ohnishi, T., Shiraishi, T., Osame, M., and Ohno, S. 2004. Inhibition of nonsensemediated mRNA decay rescues the phenotype in Ullrich's disease. Ann. Neurol. 55: 740-744.

Usuki, F., Yamashita, A., Kashima, I., Higuchi, I., Osame, M., and Ohno, S. 2006. Specific inhibition of nonsense-mediated mRNA decay components, SMG-1 or Upf1, rescues the phenotype of ullrich disease fibroblasts. Mol. Ther. 14: 351360.

Welch, E.M., Barton, E.R., Zhuo, J., Tomizawa, Y., Friesen, W.J., Trifillis, P., Paushkin, S., Patel, M., Trotta, C.R., Hwang, S., et al. 2007. PTC124 targets genetic disorders caused by nonsense mutations. Nature 447: 87-91.

Yamashita, A., Ohnishi, T., Kashima, I., Taya, Y., and Ohno, S. 2001. Human SMG-1, a novel phosphatidylinositol 3-kinaserelated protein kinase, associates with components of the mRNA surveillance complex and is involved in the regulation of nonsense-mediated mRNA decay. Genes \& Dev. 15: 2215-2228.

Yamashita, A., Kashima, I., and Ohno, S. 2005. The role of SMG-1 in nonsense-mediated mRNA decay. Biochim. Biophys. Acta 1754: 305-315. 


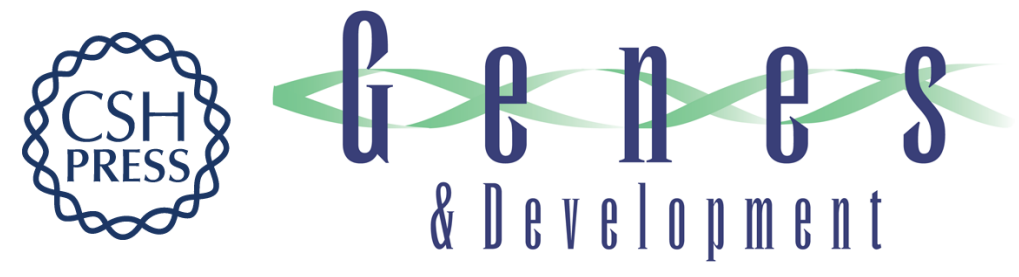

\section{SMG-8 and SMG-9, two novel subunits of the SMG-1 complex, regulate remodeling of the mRNA surveillance complex during nonsense-mediated mRNA decay}

Akio Yamashita, Natsuko Izumi, Isao Kashima, et al.

Genes Dev. 2009, 23:

Access the most recent version at doi:10.1101/gad.1767209

Supplemental Material

References License

Email Alerting Service
http://genesdev.cshlp.org/content/suppl/2009/05/08/23.9.1091.DC1

This article cites 45 articles, 20 of which can be accessed free at: http://genesdev.cshlp.org/content/23/9/1091.full.html\#ref-list-1

Receive free email alerts when new articles cite this article - sign up in the box at the top right corner of the article or click here.

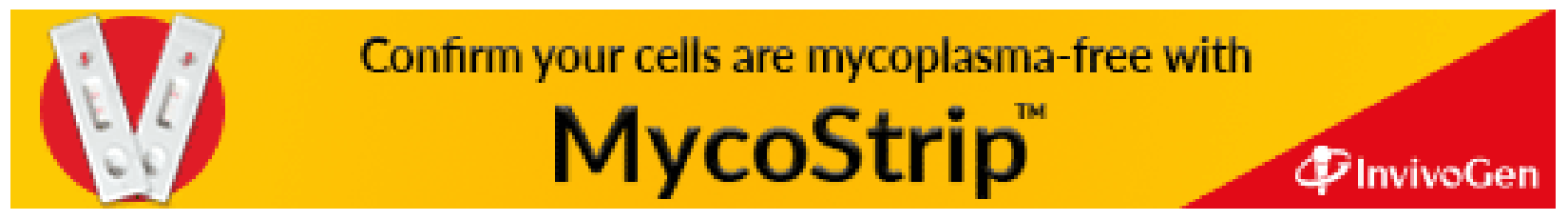

